\title{
Superposability in Hydrodynamic and MHD Flow
}

\author{
Shruti Rastogi, Brijender Nath Kaul, Sanjeev Rajan \\ Department of Mathematics, Hindu College, Moradabad, India \\ Email: shruti81rastogi@gmail.com
}

Received 14 March 2015; accepted 12 June 2015; published 15 June 2015

Copyright (C) 2015 by authors and Scientific Research Publishing Inc.

This work is licensed under the Creative Commons Attribution International License (CC BY).

http://creativecommons.org/licenses/by/4.0/

(c) (i) Open Access

\begin{abstract}
In this paper, phenomena of superposability and self superposability in hydrodynamics and magneto hydrodynamics have been discussed. One of the most important applications of superposability in hydrodynamics is the construction of exact analytic solution of the basic equation of fluid dynamics. Kapur and Bhatia have given a simple idea that if two velocity vectors have self superposable and mutually superposable motion then sum or difference of these two is self superposable and vice versa and if each of the vector is superposable on the third then their sum and difference are also superposable on the third. For superposability in magneto-hydrodynamics many mathematicians like Ram Moorthy, Ram Ballabh, Mittal, Kapur \& Bhatia and Gold \& Krazyblocki have defined it in various ways, especially Kapur \& Bhatia generalized the well-known work on superposability by Ram Ballabh to the case of viscous incompressible electrically conducting fluids in the presence of magnetic field. We found the relationship of two basic vectors for two important curvilinear coordinate systems for their use in our work. We've found the equations of div, curl and grad for a unit vector in parabolic cylinder coordinates and ellipsoidal coordinates for further use.
\end{abstract}

\section{Keywords}

Superposability, Hydrodynamics and Magneto-Hydrodynamics, MHD Flow

\section{Introduction}

It was shown by Ballabh [1] that when a flow with velocity $\bar{q}$ satisfied the condition:

$$
\operatorname{curl}(\bar{q} \times \operatorname{curl} \bar{q})=0,
$$

it became self superposable. In the present chapter, the authors have attempted some fluid velocities for which $(\bar{q} \times \operatorname{curl} \bar{q})=\bar{p}$ (say) can be represented as the gradient of a scalar quantity. Attention has been focused on in- 
compressible fluids only. For such fluids some velocities have been determined which satisfy the continuity condition in parabolic cylinder system of coordinates [2] and for which $\bar{p}$ can be represented as the gradient of a scalar quantity $\theta$ (say). The solutions thus found must be of self superposable type. Each will have a set of constants which can be determined by the boundary conditions.

It has further shown by Ballabh [3] that if $q_{1}$ and $q_{2}$ are self superposable flows and $q_{1}+q_{2}$ is also selfsuperposable then $q_{1}$ and $q_{2}$ are mutually superposable. By using this property some more self superposable flows have been determined. Pressure distribution for some of the flows has also been attempted. Attempts have also been made to find some curves along which the vorticity of the flow becomes constant and also the conditions of irrotationality. P. K. Mittal, V. Singh and Sanjeev Rajan [4] have also analyzed vorticity of hydromagnetic converting slip flow through a horizontal chanel.

In this paper, a method will be introduced to solve the equations of fluid dynamics in parabolic cylinder coordinates by using the property of self superposable. Mittal [5], Mittal et al. [6]-[9] have solved the equations for different coordinate systems.

\section{Hydrodynamic Superposability}

Due to non-linearity of the equations of motion in the theory of fluid dynamics we are frequently compelled to resort to approximate methods of solutions which depend on the assumption that certain terms, usually the non-linear terms are small compared with those retained so that the solutions obtained are valid only when the motion is slow per leaps, equally as often it is assumed that two or more distinct motions are linearly superposable. In some cases both assumptions are made.

The idea of superposability as regards fluid motion does not seem to have engaged the attention of mathematicians in a formal way until the year 1940, when Ram Ballabh [10] published his first paper dealing with the subject. Earlier workers merely assumed that in a homogeneous incompressible fluid moving irrotationally under conservative forces, the result of superimposing the velocity on the other is just to add the two. The difficulty arises when one of the motions at least is not irrotational.

A mathematical definition of superposability of two solutions the hydro dynamical equations governing the motion of a viscous homogeneous incompressible fluid as given by Ram Ballabh as follows:

Let $q_{1}^{\prime}, q_{2}^{\prime}$ be the velocity vectors of the flows each satisfying the equation of motion corresponding to given (but not necessarily the same) external forces, initial conditions, boundaries and boundary conditions.

$$
\frac{\partial q^{\prime}}{\partial t}-q \times \operatorname{curl} q=F^{\prime} \nabla\left(\frac{p}{\rho}+\frac{1}{2} q^{2}\right)+v \rho^{2} q^{\prime}
$$

Let $p_{1}, p_{2}$ be the corresponding pressures and $F_{1}, F_{2}$ the corresponding external forces.

The two flows are said to be superposable if a pressure $p_{1}+p_{2}+\pi$ can be determined so that the fluid moves with the velocity $q_{1}^{\prime}+q_{2}^{\prime}$ under the external forces $F_{1}+F_{2}$ with the necessary modifications in the initial and boundary conditions.

This is not the only possible definition of superposability, we might, for example assume that $\left(q_{1}^{\prime}, p_{1}\right)$, $\left(q_{2}^{\prime}, p_{2}\right)$ and $\left(q_{1}^{\prime}+q_{2}^{\prime}, p_{1}+p_{2}+\pi\right)$ should satisfy the equations of motion under the same external forces is conservative.

Substituting $\left(q_{1}^{\prime}, p_{1}\right),\left(q_{2}^{\prime}, p_{2}\right)$ and $\left(q_{1}^{\prime}+q_{2}^{\prime}, p_{1}+p_{2}+\pi\right)$ in (1.1) and eliminating $\pi$ we get,

$$
\operatorname{curl}\left(q_{1}^{\prime} \times \operatorname{curl} q_{2}^{\prime}+q_{2}^{\prime} \times \operatorname{curl} q_{1}^{\prime}\right)=0
$$

as the condition of mutual superposability of $q_{1}^{\prime}$ and $q_{2}^{\prime}$.

In the motion $q^{\prime}$ is self superposable, i.e. superposable on itself, Equation (1.2) simplifies to

$$
\operatorname{curl}\left(q_{1}^{\prime} \times \operatorname{curl} q_{2}^{\prime}\right)=0 .
$$

The definition of superposability can be generalized so as to include homogeneous viscous incompressible fluids of kinematic viscosities $v_{1}, v_{2}$ and $v$, this was done by Truesdall [11] in 1954 and his condition of superposability of $q_{1}^{\prime} \& q_{2}^{\prime}$ is $\left(v-v_{1}\right)$.

$$
\operatorname{curlcurl}\left(\operatorname{curl} q_{1}^{\prime}\right)+\left(v_{1}-v_{2}\right) \operatorname{curlcurl}\left(\operatorname{curl} q_{2}^{\prime}\right)-\operatorname{curl}\left(q_{1}^{\prime} \times \operatorname{curl} q_{2}^{\prime}+q_{2}^{\prime} \times \operatorname{curl} q_{1}^{\prime}\right)=0 \text {. }
$$

Gold and Krazyblocki [12] [13] extended the definition of superposability to non-Newtonian and compressi- 
ble fluids, in each case of non-Newtonian fluids they introduced a corresponding scalar function $\pi$ for each nonlinear terms resulting in an additional equation added $\pi$. Swati Gupta and Sanjeev Rajan [14] also explored heat transfer of non-Newtonian fluid in porous medium.

In 1965 Kapur and Bhatia [15] modified the definition of superposability as follows:

If $q_{1}^{\prime}$ and $q_{2}^{\prime}$ are two possible velocity vector fields (i.e. vectors satisfying the basic equation determining the motion of the system), then these will said to be superposable additive if $q_{1}^{\prime}+q_{2}^{\prime}$ is also a possible velocity vector field.

Kapur and Bhatia made the following remarks about their definition:

1) It is not restricted to incompressible flows, i.e. it can be applied equally well to compressible flows.

2) It is not restricted to Newtonian flows and it can be applied equally well to non-Newtonian flows.

3) It is implicit in this definition that initial and boundary condition may have to be modified that pressure field may have to modify and for compressible flows, even density, temperature and entropy fields may have to be modified.

4) It is not necessary to speak of superposed motion only under a force system which is vector sum of the two force systems.

One of the important applications of the principle of superposability is in the construction of exact analytic solutions of the basic equations of fluid dynamics. The object is not so much to solve practical problems as to obtain special solutions of the basic equations without reference to the boundary conditions. Since these basic equations form the basic literally tens of thousands of research papers, even some special solutions may be of same interest.

The underlying principle is very simple. Suppose we know a solution $\left(u_{1}, v_{1}, w_{1}\right)$ of the basic equations. We attempt to find other solutions $\left(u_{2}, v_{2}, w_{2}\right)$ such that $\left(u_{1}+u_{2}, v_{1}+v_{2}, w_{1}+w_{2}\right)$ is also a solution of the basic equations. The basic fluid dynamics equation will determine differential equations to determine $\left(u_{2}, v_{2}, w_{2}\right)$.

The following special cases are of special interest:

\begin{tabular}{cccc}
\hline & Original solution & Solution added & Superposed solution \\
\hline 1 & $\left(u_{1}, v_{1}, 0\right)$ & $\left(u_{2}, v_{2}, 0\right)$ & $\left(u_{1}+u_{2}, v_{1}+v_{2}, 0\right)$ \\
2 & $(u, v, 0)$ & $(0,0, w)$ & $(u, v, w)$ \\
3 & $\left(u_{1}, 0, u_{2}\right)$ & $\left(0, u_{0}, 0\right)$ & $\left(u_{1}, u_{0}, u_{2}\right)$ \\
4 & $\left(0, u_{0}, 0\right)$ & $\left(u_{1}, 0, u_{2}\right)$ & $\left(u_{1}, u_{0}, u_{2}\right)$ \\
\hline
\end{tabular}

It is obvious that for our purpose, it is not necessary to insist that $\left(u_{2}, v_{2}, w_{2}\right)$ be a solution of the basic equations, what is important is that $\left(u_{1}+u_{2}, v_{1}+v_{2}, w_{1}+w_{2}\right)$ should be a solution. This simple idea has been exploited by Kapur and Bhatia to obtain more general solutions that were available earlier.

Some properties:

It is easy to establish the following theorems using Equations (1.2) and (1.3).

1) If $q_{1}^{\prime}, q_{2}^{\prime}$ are two self superposable and mutually superposable motion then $q_{1}^{\prime} \pm q_{2}^{\prime}$ are self superposable.

2) If $q_{1}^{\prime}, q_{2}^{\prime}$ are self superposable and $q_{1}^{\prime} \pm q_{2}^{\prime}$ are also self superposable then $q_{1}^{\prime}$ and $q_{1}^{\prime}$ are mutually superposable.

3) If each of $q_{1}^{\prime}$ and $q_{2}^{\prime}$ is superposable on a third flow $q_{3}^{\prime}$, then $q_{1}^{\prime} \pm q_{2}^{\prime}$ are also superposable on the flow $q_{3}^{\prime}$.

\section{Superposability in Magnetohydrodynamics}

Kapur [16] [17] and Bhatnagar [18] generalized the well-known work on superposability by Ram Ballabh to the case of viscous incompressible electrically conducting fluids is the presence of magnetic field. The three different definitions of superposability in magnetohydrodynamics are as follows:

\subsection{Definition 1 (Kapur) [16] [17]}

Two incompressible hydromagnetic motions with uniform density $\rho$, velocity vectors $q_{1}^{\prime}, q_{2}^{\prime}$; pressures $p_{1}, p_{2}$; 
electric intensities $E_{1}^{\prime}, E_{2}^{\prime}$; current density vectors $J_{1}^{\prime}, J_{2}^{\prime}$; magnetic fields $H_{1}^{\prime}, H_{2}^{\prime}$; and force potentials $\Omega_{1}^{\prime}, \Omega_{2}^{\prime}$ are said to be superposable if these exists a flow $q_{1}^{\prime}+q_{2}^{\prime}, p_{1}+p_{2}+\pi, E_{1}^{\prime}+E_{2}^{\prime}, J_{1}^{\prime}+J_{2}^{\prime}, H_{1}^{\prime}+H_{2}^{\prime}$, $\Omega_{1}^{\prime}+\Omega_{2}^{\prime}$ satisfying the basic equation of magnetohydrodynamics when each one of them satisfies separately.

A set of necessary and sufficient conditions for these are:

$$
\operatorname{curl}\left(\omega_{2}^{\prime} \times q_{1}^{\prime}+\omega_{1}^{\prime} \times q_{2}^{\prime}\right)=\frac{\mu_{e}}{\rho}\left(J_{2}^{\prime} \times H_{1}^{\prime}+J_{1}^{\prime} \times H_{2}^{\prime}\right)
$$

and

$$
\left(q_{2}^{\prime} \times H_{1}^{\prime}+q_{1}^{\prime} \times H_{2}^{\prime}\right)=0
$$

and then $\pi$ is given by:

$$
\operatorname{grad} \pi=-\rho\left[\operatorname{grad}\left(q_{1}^{\prime} \cdot q_{2}^{\prime}\right)+\omega_{2}^{\prime} \times q_{1}^{\prime}+\omega_{2}^{\prime} \times q_{1}^{\prime}\right]-\frac{\mu_{e}}{\rho}\left(J_{2}^{\prime} \times H_{1}^{\prime}+J_{1}^{\prime} \times H_{2}^{\prime}\right) .
$$

\subsection{Definition 2 (Bhatnagar) [18]}

The incompressible hydromagnetic motion $q_{i}^{\prime}, p_{i}, E_{i}^{\prime}, J_{i}^{\prime}, H_{i}^{\prime}, \Omega_{i}^{\prime}(i=1,2)$ are said to be superposable, if these exists a flow

$$
q_{1}^{\prime}+q_{2}^{\prime}, p_{1}+p_{2}+\pi, E_{1}^{\prime}+E_{2}^{\prime}+\operatorname{grad}, J_{1}^{\prime}+J_{2}^{\prime}, H_{1}^{\prime}+H_{2}^{\prime}, \Omega_{1}^{\prime}+\Omega_{2}^{\prime}
$$

satisfying the basic equations of magnetohydrodynamics, when each one of them satisfies those separately.

A set of necessary and sufficient conditions for these are given by (1.5) and

$$
\operatorname{curl}\left(q_{1}^{\prime} \times H_{2}^{\prime}+q_{2}^{\prime} \times H_{1}^{\prime}\right)=0
$$

and then $\operatorname{grad} \pi$ and $\operatorname{grad} \phi$ are respectively given by (1.7) and

$$
\operatorname{grad} \phi=-\frac{\mu_{e}}{\rho}\left(q_{1}^{\prime} \times H_{2}^{\prime}+q_{2}^{\prime} \times H_{1}^{\prime}\right) .
$$

\subsection{Definition 3 (Gold and Krazyblocki) [12] [13]}

If $u_{i}^{\prime}$ and $h_{i}^{\prime}$ denote the velocity and magnetic field components respectively, then two fields $u^{\prime \prime}, h_{i}^{\prime \prime}, u_{i}^{\prime \prime \prime}$, $h_{i}^{\prime \prime \prime}$ are said to be superposable, if there is a scalar function $\pi$ such that

$$
-\left(u_{i}^{\prime \prime} u_{k}^{\prime \prime \prime}+u_{k}^{\prime \prime} u_{i}^{\prime \prime \prime}-h_{i}^{\prime \prime} h_{k}^{\prime \prime \prime}-h_{k}^{\prime \prime} h_{i}^{\prime \prime \prime}\right)_{{ }_{k}}=-\pi, i
$$

where comma followed by a suffix denotes differentiation with respect to the variable corresponding to that suffix.

Note: in above definitions $q^{\prime}, \omega^{\prime}, H^{\prime}, E^{\prime}, J^{\prime}$ are velocity, vorticity, magnetic field, electric intensity and current density vectors respectively and $p, \rho, \Omega, \mu_{e}, v$ and $\sigma$ are the pressure, density, force potential, magnetic permeability, kinematic viscosity coefficient and conductivity respectively.

Kapur [15] [19]-[21] made the following remarks on the above definitions:

1) The first condition in each of three cases is the same, but differs in notation.

2) To make the distinction between the definitions 1 and 2. Let us say that the two fields are definition. It is obvious that two fields, which are strictly superposable, are also superpo "superposable" if they satisfy Bhatnagar's definition and strictly superposable if they satisfy Kapur's table but if the two fields are superposable they may not be strictly superposable. For more superposability, we want $H^{\prime}, q^{\prime}$ (and also $J^{\prime}$ ) to be active.

3) Gold and Krazyblocki do not give any second condition and their statement that they are assuming $H^{\prime}$ to be another linear property is only partially correct. Since they use this property while dealing with equation of momentum but they do not use it in correction with magnetic function equation. Their condition (1.11) therefore, ensures that only partially superposability, it has to be supplemented by:

$$
\left(h_{i}^{\prime \prime} u_{k}^{\prime \prime \prime}-u_{i}^{\prime \prime} h_{k}^{\prime \prime \prime}+h_{i}^{\prime \prime \prime} u_{k}^{\prime \prime}-u_{i}^{\prime \prime \prime} h_{k}^{\prime \prime}\right)_{, k}=0
$$

and for strict superposability, it has to be supplement by 


$$
\varepsilon_{i j k}\left(u_{j}^{\prime \prime} h_{k}^{\prime \prime \prime}+u_{j}^{\prime \prime \prime} h_{k}^{\prime \prime}\right)=0
$$

where $\varepsilon_{i j k}$ is the alternative tensor.

In addition to introducing the concept of strict superposability to deal with non-linear terms in basic equations by hydromagnetic. Kapur [21] has also obtained superposability conditions both in case of two dimensional and axially symmetric superposable flows by assuming solenoidal, velocity and magnetic field vectors to be two dimensional and in second case having both poloridal and toroidal components. Chandrashekhar's [22] equation for axially symmetric flows has been extended to viscous fields. It has been shown further that force free fields, (i.e. $\operatorname{curl} H \times H=0$ ) and self superposable flows are particular case of this concept.

In continuation his paper Kapur [16] discussed:

1) Superposability of wave motion.

2) Hydrostatic equilibrium of magnetic stars.

3) Effects of viscosity on axially symmetric hydromagnetic flows.

4) Axially symmetric force free fields and

5) General force free fields.

Teeka Rao [23] also obtained:

1) Necessary and sufficient conditions for hydromagnetic superposable flows.

2) Superposability conditions in each case of both two dimensional and axially symmetric superposable flows with solenoidal velocity and magnetic field.

3) Superposability conditions by assuming velocity and magnetic fields to be having poloridal components only.

Ram Moorthy [24] proved two theorems in case of axially symmetric hydromagnetic flows. Kapur [20] generalized one of the theorems proved by Ram Moorthy and proved one more theorem also. Bhatnagar [18] in addition to discussion of superposability in hydromagnetic flows also discussed the harmonic analysis of general spatial motion with magnetic field. Mittal [25] used the phenomenon of superposability and that of self-superposability in the case of a magneto hydrodynamic flow in steady state under a force free magnetic field.

Mittal et al. have studied the superposability and self-superposability of a number of duct flows and have used the phenomenon in studying the flows in some non-customary type of tubes and cross sections viz.

1) Steady laminar magneto hydrodynamic flow [26].

2) Flow between two co-axial rotating cylinders in a radial magnetic field.

3) Stationary flow of a conducting liquid in an infinitely long annular tube in presence of radial magnetic field.

4) MHD flow in a rectangular duct [27].

5) MHD flow in elliptic cylinder coordinate system.

6) Self-superposable flows in conical ducts [7].

7) Self-superposable fluid motions in toroidal ducts [28].

8) MHD flows over conducting walls.

9) Self-superposable motions in paraboloidal ducts [8] [19] [29].

10) Self-superposable flows in ducts having confocal ellipsoidal shape [30].

Mittal [25] also studied some features of two dimensional magnetohydrodynamic flows, in steady state and under force free magnetic fields, by using the phenomenon of superposability and self-superposability. Shruti Rastogi, B. N. Kaul and Sanjeev Rajan [31] have taken the work of Mittal et al. further. In their work they discussed about some magnetic fields with conservative Lorentz force in confocal paraboloidal coordinates. They showed that under some conditions magnetohydrostatic configuration may be formed by self-superpoable flow of an electrically conducting incompressible fluid permeated by a magnetic field with conservative Lorentz force. V. Singh and S. Rajan [32] have extended Mittal's work further in Ellipsoidal ducts.

Kapur [20] discussed properties of force free fields by proving some theorems. The conditions for hydromagnetic flow to be self superposable are:

$$
\begin{gathered}
\operatorname{curl}\left(q^{\prime} \times \operatorname{curl} q^{\prime}\right)=\frac{\mu_{e}}{\rho}\left(H^{\prime} \times \operatorname{curl} H^{\prime}\right) \\
\left(q^{\prime} \times H^{\prime}\right)=0 \text {, i.e. } q^{\prime}=H^{\prime} .
\end{gathered}
$$

Kapur [21] discussed the characterization of 
1) Most general axi-symmetric self-superposable flows with (a) constant $\alpha$ (b) variable $\alpha$ and

2) Most general axi-symmetric force free hydromagnetic flows.

Bhatia [2] discussed axially symmetric self-superposable hydromagnetic superposable flows. The most general steady, axially symmetric flows of the following types have been obtained.

1) Poloridal velocity fields with toroidal magnetic field and toroidal velocity field with poloridal magnetic field superposable on each other.

2) Axi symmetric velocity field with toroidal magnetic field and toroidal velocity field with axi symmetric magnetic field superposable on each other, when the first flow has no radial velocity component and the second flow has no radial magnetic field component.

3) Toroidal velocity fields with toroidal magnetic fields superposable on each other. When the conditions of integrability are satisfied in case of both flows to be superposed.

4) Axi symmetric solutions of the basic equations of magnetohydrodynamic form.

a) Poloridal velocity field having no radial component with toroidal magnetic field.

b) Toroidal velocity field with poloridal magnetic field where we require only one of the flows to satisfy the conditions of integrability.

\section{Curvilinear Coordinates}

\subsection{Unitary and Reciprocal Unitary Vectors}

In a given region of space, we may associate with each point of the region the Cartesian coordinates $(x, y, z)$. This description of the points of space is unique so long as we restrict ourselves to the given Cartesian system. However, in the region of space, we can define three independent, single valued functions of the Cartesian set:

$$
u=f_{1}(x, y, z), v=f_{2}(x, y, z), w=f_{3}(x, y, z) .
$$

If we consider a particular point in the region, say $P\left(x_{0}, y_{0}, z_{0}\right)$, we can associate with such point the three functional values $u_{0}, v_{0}, w_{0}$ which are obtained by setting $x, y, z$ equal to $x_{0}, y_{0}$ and $z_{0}$ respectively.

Under very general conditions we can solve the set of Equations (1.16) to obtain

$$
x=g_{1}(u, v, w), \quad y=g_{2}(u, v, w), z=g_{3}(u, v, w)
$$

where the function $g_{1}, g_{2}, g_{3}$ are also independent and continuous functions. Generally, these functions are not single valued for the entire range of $u, v$ and $w$. Thus for each triplet of numbers $u, v$ and $w$, there will generally correspond one and only one point $P(x, y, z)$ in the given region of space, therefore a one to one correspondence between the triplet $(u, v, w)$ and the points of a region of space. The set of functions $(u, v, w)$ can be termed a set of coordinated for the points in space. These coordinates are generally known as generalized or curvilinear coordinates.

Though each point $P$ in the given region of space, these will pass the three surfaces.

$$
u=\text { constant, } v=\text { constant, } w=\text { constant }
$$

which are known as the coordinate surfaces. Any two of these constant surfaces intersect in a space curve. The set of three curves through the point $P$ is known as the coordinate curves of the point $P$. As a matter of nomenclature, we shall adopt the following convention to distinguish between the coordinate curves:

$$
\left.\begin{array}{l}
u=\text { coordinate curve }, v=\text { constant }, w=\text { constant } \\
v=\text { coordinate curve }, w=\text { constant }, u=\text { constant } \\
w=\text { coordinate curve }, u=\text { constant }, v=\text { constant }
\end{array}\right\}
$$

The coordinate surfaces and coordinate curves associated with the point $P$ are given in Figure 1.

If $\bar{r}$ is the radius vector from an arbitrary origin to the point $P$, it can be regarded as a function of a generalized coordinates of the point.

$$
\bar{r}=\bar{r}(u, v, w)
$$

The change in the radius vector $\bar{r}$ due to infinitesimal displacements along the three coordinate curves is

$$
\mathrm{d} \bar{r}=\frac{\partial \bar{r}}{\partial u} \mathrm{~d} u+\frac{\partial \bar{r}}{\partial v} \mathrm{~d} v+\frac{\partial \bar{r}}{\partial w} \mathrm{~d} w
$$




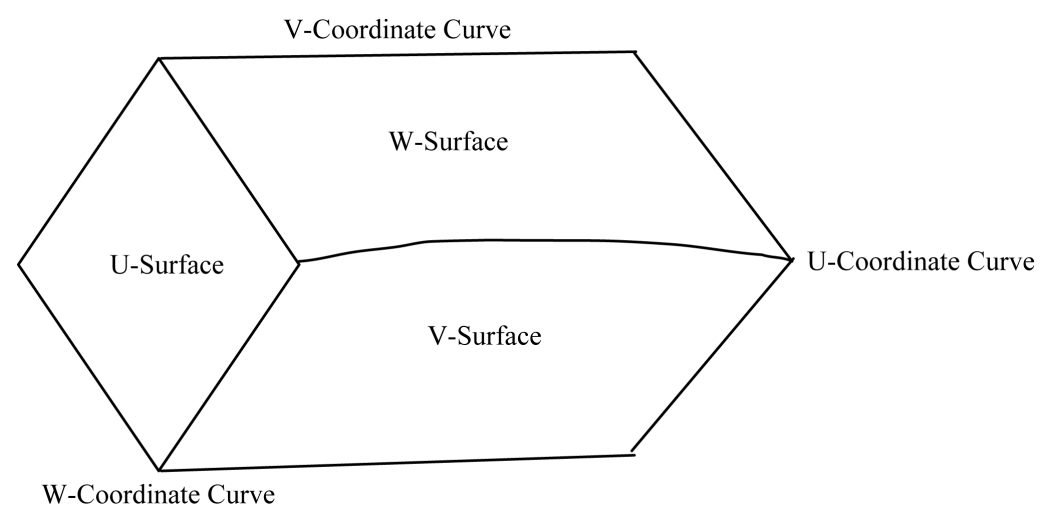

Figure 1. Coordinate surfaces and coordinate curve.

Thus, the change in $\bar{r}$ due to a unit displacement along the $u$-coordinate curve is $\frac{\partial \bar{r}}{\partial u}$. Similarly, unit displacement along the $v$ and $w$-coordinate curves induce the changes $\frac{\partial \bar{r}}{\partial v}$ and $\frac{\partial \bar{r}}{\partial w}$ respectively. We define the set of vectors:

$$
\bar{e}_{1}=\frac{\partial \bar{r}}{\partial u}, \bar{e}_{2}=\frac{\partial \bar{r}}{\partial v}, \bar{e}_{3}=\frac{\partial \bar{r}}{\partial w}
$$

which are known as the unitary vectors associated with the point $P$. It is to be noted that those vector are not generally of unit length, and that their dimensions depend on the nature of the generalized coordinates. They do, however, serve as a base of reference in the sense that any vector whose initial point is at $P$ can be expressed as a linear combination of the set of unitary vectors.

In particular

$$
\mathrm{d} \bar{r}=\bar{e}_{1} \mathrm{~d} u+\bar{e}_{2} \mathrm{~d} v+\bar{e}_{3} \mathrm{~d} w .
$$

Since the set of unitary vectors are non-coplanar, they define a parallelepiped whose volume is given by

$$
V=\bar{e}_{1} \cdot\left(\overline{e_{2}} \times \bar{e}_{3}\right)=\overline{e_{2}} \cdot\left(\overline{e_{3}} \times \overline{e_{1}}\right)=\bar{e}_{3} \cdot\left(\overline{e_{1}} \times \bar{e}_{2}\right) .
$$

We may define triplet of vectors, known as the reciprocal unitary vectors by the relation

$$
\bar{e}_{1}^{-1}=\frac{\bar{e}_{2} \times \bar{e}_{3}}{V}, \bar{e}_{2}^{-1}=\frac{\bar{e}_{3} \times \bar{e}_{1}}{V}, \bar{e}_{3}^{-1}=\frac{\bar{e}_{1} \times \bar{e}_{2}}{V} .
$$

It is clear that from the definition $e^{-1}$ is normal to the plane defined by the unitary vectors $\left(\bar{e}_{2}, \bar{e}_{3}\right)$. Similarly $e^{-2}$ is normal to the plane of $\left(\bar{e}_{3}, \bar{e}_{1}\right)$ and $e^{-3}$ is normal to the plane of $\left(\bar{e}_{1}, \bar{e}_{2}\right)$, then it follows that

$$
\left.\begin{array}{l}
\bar{e}_{1}^{-1} \cdot \bar{e}_{2}=\bar{e}_{1}^{-1} \cdot \bar{e}_{3}=0, \bar{e}_{1}^{-1} \cdot \bar{e}_{1}=1 \\
\bar{e}_{2}^{-1} \cdot \bar{e}_{3}=\bar{e}_{2}^{-1} \cdot \bar{e}_{1}=0, \bar{e}_{2}^{-1} \cdot \bar{e}_{2}=1 \\
\bar{e}_{3}^{-1} \cdot \bar{e}_{1}=\bar{e}_{3}^{-1} \cdot \bar{e}_{2}=0, \bar{e}_{3}^{-1} \cdot \bar{e}_{3}=1
\end{array}\right\}
$$

The set of nine Equations (1.26) is usually written

$$
\bar{e}_{i}^{-1} \cdot \bar{e}_{j}=\delta_{j}^{i}
$$

where the symbol $\delta_{j}^{i}$ known as kroneckar delta, is defined by

$$
\delta_{j}^{i}= \begin{cases}1, & i=j \\ 0, & i \neq j\end{cases}
$$

The set of reciprocal unitary vectors also form a basis for the point $P$ in the same sense as the unitary vectors. The relation between the unitary vectors and the reciprocal unitary vectors is known in Figure 2. 


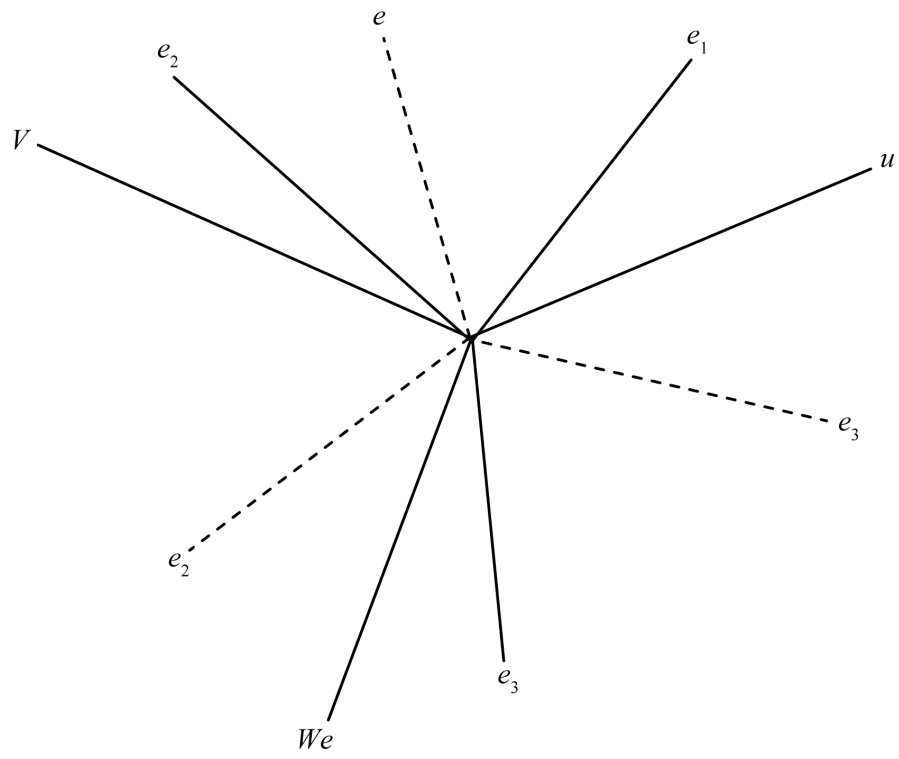

Figure 2. Relation between the unitary vectors and the reciprocal unitary vectors.

In terms of the reciprocal unitary vectors, the differential $\mathrm{d} \bar{r}$ has the representation:

$$
\mathrm{d} \bar{r}=\bar{e}_{1}^{-1} \mathrm{~d} u^{\prime}+\bar{e}_{2}^{-1} \mathrm{~d} v^{\prime}+\bar{e}_{3}^{-1} \mathrm{~d} w^{\prime} .
$$

The differentials $\mathrm{d} u^{\prime}, \mathrm{d} v^{\prime}$ and $\mathrm{d} w^{\prime}$ are obviously the components of $\mathrm{d} \bar{r}$ in the direction specified by the reciprocal unitary vectors and are functions of the generalized coordinates $(u, v, w)$. However, these differentials are related of the generalized coordinates by a set of linear equations which are not in general integrable. If we equate the representations of $d \bar{r}$ in terms of unitary and reciprocal unitary vectors we have:

$$
\mathrm{d} \bar{r}=\bar{e}_{1} \mathrm{~d} u+\bar{e}_{2} \mathrm{~d} v+\bar{e}_{3} \mathrm{~d} w=\bar{e}_{1}^{-1} \mathrm{~d} u^{\prime}+\bar{e}_{2}^{-1} \mathrm{~d} v^{\prime}+\bar{e}_{3}^{-1} \mathrm{~d} w^{\prime} .
$$

Now we form the inner product of $\mathrm{d} \bar{r}$ successfully with $\bar{e}^{-1}, \bar{e}^{-2}$ and $\bar{e}^{-3}$ and use the orthogonality relations to obtain the set of equations:

$$
\left.\begin{array}{l}
\mathrm{d} u=\bar{e}_{1}^{-1} \bar{e}_{1}^{-1} \mathrm{~d} u^{\prime}+\bar{e}_{1}^{-1} \bar{e}_{2}^{-1} \mathrm{~d} v^{\prime}+\bar{e}_{1}^{-1} \bar{e}_{3}^{-1} \mathrm{~d} w^{\prime} \\
\mathrm{d} v=\bar{e}_{2}^{-1} \bar{e}_{1}^{-1} \mathrm{~d} u^{\prime}+\bar{e}_{2}^{-1} \bar{e}_{2}^{-1} \mathrm{~d} v^{\prime}+\bar{e}_{2}^{-1} \bar{e}_{3}^{-1} \mathrm{~d} w^{\prime} \\
\mathrm{d} w=\bar{e}_{3}^{-1} \bar{e}_{1}^{-1} \mathrm{~d} u^{\prime}+\bar{e}_{3}^{-1} \bar{e}_{2}^{-1} \mathrm{~d} v^{\prime}+\bar{e}_{3}^{-1} \bar{e}_{3}^{-1} \mathrm{~d} w^{\prime}
\end{array}\right\}
$$

Similarly, if we take the inner product of $\mathrm{d} \bar{r}$ with $\bar{e}_{1}, \bar{e}_{2}$ and $\bar{e}_{3}$ we obtain the differential equations:

$$
\left.\begin{array}{l}
\mathrm{d} u^{\prime}=\bar{e}_{1} \cdot \bar{e}_{1} \mathrm{~d} u+\bar{e}_{1} \cdot \bar{e}_{2} \mathrm{~d} v+\overline{e_{1}} \cdot \bar{e}_{3} \mathrm{~d} w \\
\mathrm{~d} v^{\prime}=\bar{e}_{2} \cdot \bar{e}_{1} \mathrm{~d} u+\bar{e}_{2} \cdot \bar{e}_{2} \mathrm{~d} v+\bar{e}_{2} \cdot \bar{e}_{3} \mathrm{~d} w \\
\mathrm{~d} w^{\prime}=\bar{e}_{3} \cdot \bar{e}_{1} \mathrm{~d} u+\bar{e}_{3} \cdot \bar{e}_{2} \mathrm{~d} v+\bar{e}_{3} \cdot \bar{e}_{3} \mathrm{~d} w
\end{array}\right\}
$$

It is convenient to represent the inner product of the unitary vectors, and the reciprocal unitary vectors by the set of quantities:

$$
\left.\begin{array}{l}
g^{i j}=\bar{e}_{i}^{-1} \cdot \bar{e}_{j}^{-1} g^{j i} \\
\bar{g}_{i j}=\bar{e}_{j} \cdot \bar{e}_{i}=\bar{g}_{j i}
\end{array}\right\}
$$

These quantities are fundamental in the representation of the vector differential operator in terms of generalized coordinates, and in the formalism of tensor analysis. In terms of this notation, the components of $\mathrm{d} \bar{r}$ with respect to the unitary and reciprocal vectors are related by 


$$
\left.\begin{array}{l}
\mathrm{d} u=g^{11} \mathrm{~d} u^{\prime}+g^{12} \mathrm{~d} v^{\prime}+g^{13} \mathrm{~d} w^{\prime} \\
\mathrm{d} v=g^{21} \mathrm{~d} u^{\prime}+g^{22} \mathrm{~d} v^{\prime}+g^{23} \mathrm{~d} w^{\prime} \\
\mathrm{d} w=g^{31} \mathrm{~d} u^{\prime}+g^{32} \mathrm{~d} v^{\prime}+g^{33} \mathrm{~d} w^{\prime}
\end{array}\right\}
$$

and

$$
\left.\begin{array}{l}
\mathrm{d} u^{\prime}=g_{11} \mathrm{~d} u+g_{12} \mathrm{~d} v+g_{13} \mathrm{~d} w \\
\mathrm{~d} v^{\prime}=g_{21} \mathrm{~d} u+g_{22} \mathrm{~d} v+g_{23} \mathrm{~d} w \\
\mathrm{~d} w^{\prime}=g_{31} \mathrm{~d} u+g_{32} \mathrm{~d} v+g_{33} \mathrm{~d} w
\end{array}\right\}
$$

Now, consider and arbitrary vector $\bar{a}$ which has initial point at the point $\bar{p}$. This vector may be represented in terms of either the set of unitary or reciprocal unitary vectors:

$$
\bar{a}=\sum_{i=1}^{3} a^{i} \bar{e}_{i}=\sum_{i=1}^{3} \bar{a}_{i} \bar{e}_{i}^{-1}
$$

where $a_{i}$ and $\bar{a}_{i}$ are the projections of $\bar{a}$ in the direction of $\bar{e}_{i}$ and $\bar{e}^{i}$ respectively. If we form the inner product of $\bar{a}_{i}$ with $\bar{e}^{i}$ we obtain as a result of the orthogonality relations

$$
a^{i}=\bar{a} \cdot \bar{e}_{i}^{-1}
$$

similarly

$$
a_{i}=\bar{a} \cdot \bar{e}_{i} .
$$

The two sets of components of the vector space $\bar{a}$ are related to one another by

$$
a^{j}=\sum_{j=1}^{3} g^{i j} a_{i}, \quad a_{i}=\sum_{i=1}^{3} g_{j i} a^{i} .
$$

The arbitrary vector $\bar{a}$ thus has either of the representations:

$$
\bar{a}=\sum_{i=1}^{3}\left(a^{i} \bar{e}_{i}^{-1}\right) \bar{e}_{i}=\sum_{i=1}^{3}\left(\bar{a}_{i} \bar{e}_{i}\right) \bar{e}_{i}^{-1} .
$$

In the generalized coordinate system $(u, v, w)$. The quantities $a^{i}$ are known as the contra variant components of the vector $\bar{a}$ relative to the coordinate system $(u, v, w)$. Similarly the set of quantities $a_{i}$ are known as the covariant components of the vector $\bar{a}$ relative to the coordinate system $(u, v, w)$.

Since the lengths and dimensions of the unitary and reciprocal unitary vectors depends on the nature of the set of generalized coordinates. It is clear that the covariant and contra variant components of given vector do not necessarily have the same dimensions as the given vector. In order to avoid difficulties which may be introduced by this fact, it is frequently desirable to define a set of unit vectors, but which are dimensionless.

$$
\overline{i_{1}}=\frac{\bar{e}_{1}}{\sqrt{\bar{e}_{1} \cdot \bar{e}_{1}}}=\frac{\bar{e}_{1}}{g_{11}}, \overline{i_{2}}=\frac{\bar{e}_{2}}{\sqrt{\bar{e}_{2} \cdot \bar{e}_{2}}}=\frac{\bar{e}_{2}}{g_{22}}, \overline{i_{3}}=\frac{\overline{e_{3}}}{\sqrt{\bar{e}_{3} \cdot \bar{e}_{3}}}=\frac{\overline{e_{3}}}{g_{33}}
$$

In terms of this set of dimensionless unit vectors, the arbitrary vectors $\bar{a}$ has the representation:

$$
\bar{a}=A_{1} \bar{i}_{1}+A_{2} \bar{i}_{2}+A_{3} \bar{i}_{3} .
$$

The set of components $A_{i}$ have the same dimensionality as the given vector $\bar{a}$. These are known as the physical components of the vector $\bar{a}$ relative to the coordinate system $(u, v, w)$.

\subsection{Line, Surface and Volume Elements}

The vector $\overline{\mathrm{d} r}$ represents a displacement from the point $P(u, v, w)$ to the point $Q(u+\mathrm{d} u, v+\mathrm{d} v, w+\mathrm{d} w)$ we shall define:

$$
\begin{aligned}
\mathrm{d} S^{2}= & \overline{\mathrm{d} r} \cdot \overline{\mathrm{d} r}=\bar{e}_{1} \bar{e}_{1} \mathrm{~d} u \mathrm{~d} u+\bar{e}_{1} \bar{e}_{2} \mathrm{~d} u \mathrm{~d} v+\bar{e}_{1} \bar{e}_{3} \mathrm{~d} u \mathrm{~d} w+\bar{e}_{2} \bar{e}_{1} \mathrm{~d} u \mathrm{~d} v+\bar{e}_{2} \bar{e}_{2} \mathrm{~d} v \mathrm{~d} v \\
& +\bar{e}_{2} \bar{e}_{3} \mathrm{~d} v \mathrm{~d} w+\bar{e}_{3} \bar{e}_{1} \mathrm{~d} w \mathrm{~d} u+\bar{e}_{3} \bar{e}_{2} \mathrm{~d} w \mathrm{~d} v+\bar{e}_{3} \bar{e}_{3} \mathrm{~d} w \mathrm{~d} w
\end{aligned}
$$


In terms of the coefficients $g_{i j}$, this may be written

$$
\begin{aligned}
\mathrm{d} S^{2}= & g_{11} \mathrm{~d} u \mathrm{~d} u+g_{12} \mathrm{~d} u \mathrm{~d} v+g_{13} \mathrm{~d} u \mathrm{~d} w+g_{21} \mathrm{~d} v \mathrm{~d} u+g_{22} \mathrm{~d} v \mathrm{~d} v \\
& +g_{23} \mathrm{~d} v \mathrm{~d} w+g_{31} \mathrm{~d} w \mathrm{~d} u+g_{32} \mathrm{~d} w \mathrm{~d} v+g_{33} \mathrm{~d} w \mathrm{~d} w
\end{aligned}
$$

We may also express the quantity $\mathrm{d} S^{2}$ in terms of the reciprocal unitary vectors by:

$$
\begin{aligned}
\mathrm{d} S^{2}= & g^{11} \mathrm{~d} u^{\prime} \mathrm{d} u^{\prime}+g^{12} \mathrm{~d} u^{\prime} \mathrm{d} v^{\prime}+g^{13} d u^{\prime} d w^{\prime}+g^{21} \mathrm{~d} v^{\prime} \mathrm{d} u^{\prime}+g^{22} \mathrm{~d} v^{\prime} \mathrm{d} v^{\prime} \\
& +g^{23} \mathrm{~d} v^{\prime} \mathrm{d} w^{\prime}+g^{31} \mathrm{~d} w^{\prime} \mathrm{d} u^{\prime}+g^{32} \mathrm{~d} w^{\prime} \mathrm{d} v^{\prime}+g^{33} \mathrm{~d} w^{\prime} \mathrm{d} w^{\prime}
\end{aligned}
$$

The $g_{i j}$ and $g^{i j}$ appear as the coefficients of two differential quadratic forms which express the square of the arc length in the space of the generalized coordinates $(u, v, w)$ or the related coordinates $\left(u^{\prime}, v^{\prime}, w^{\prime}\right)$.

Now, let $\mathrm{d} S_{1}$ represent an infinitesimal displacement along the $u$-coordinate curve from the point $P(u, v, w)$. Then

$$
\overline{\mathrm{d} S_{1}}=\overline{e_{1}} \mathrm{~d} u, \mathrm{~d} S_{1}=\left|\overline{\mathrm{d} S_{1}}\right|=\sqrt{g_{11}} \mathrm{~d} u
$$

Similarly, the length of arc along the $v$-coordinate and $w$-coordinate curves are given by

$$
\overline{\mathrm{d} S_{2}}=\sqrt{g_{22}} \mathrm{~d} v, \overline{\mathrm{d} S_{3}}=\sqrt{g_{33}} \mathrm{~d} w
$$

respectively.

Let us consider and infinitesimal elemental of the $u$-coordinate surface, which is bounded by intersecting $v$ coordinate and $w$-coordinate curves as shown in Figure 3.

The area of this element is

$$
\mathrm{d} a_{1}=\left|\mathrm{d} S_{2} \times \mathrm{d} S_{3}\right|=\left|\bar{e}_{2} \times \bar{e}_{3}\right| \mathrm{d} v \mathrm{~d} w=\sqrt{\left\{\left(\bar{e}_{2} \times \bar{e}_{3}\right) \cdot\left(\bar{e}_{2} \times \bar{e}_{3}\right)\right\}} \mathrm{d} v \mathrm{~d} w .
$$

Since,

$$
\left(\bar{e}_{2} \times \bar{e}_{3}\right) \cdot\left(\bar{e}_{2} \times \bar{e}_{3}\right)=\left(\bar{e}_{2} \times \bar{e}_{2}\right)\left(\bar{e}_{3} \times \bar{e}_{3}\right)-\left(\bar{e}_{2} \cdot \bar{e}_{3}\right)\left(\bar{e}_{2} \cdot \bar{e}_{3}\right)=g_{22} g_{33}-g_{23}^{2} .
$$

Hence, the elemental areas in the $u$-coordinate surface are:

$$
\mathrm{d} a_{1}=\sqrt{g_{22} g_{33}-g_{23}^{2}} \mathrm{~d} v \mathrm{~d} w .
$$

Similarly, the elemental areas in the $v$-coordinate and $w$-coordinate surfaces are given by

$$
\mathrm{d} a_{2}=\sqrt{g_{11} g_{33}-g_{13}^{2}} \mathrm{~d} u \mathrm{~d} w
$$

and

$$
\mathrm{d} a_{3}=\sqrt{g_{11} g_{22}-g_{12}^{2}} \mathrm{~d} u \mathrm{~d} v
$$

respectively.

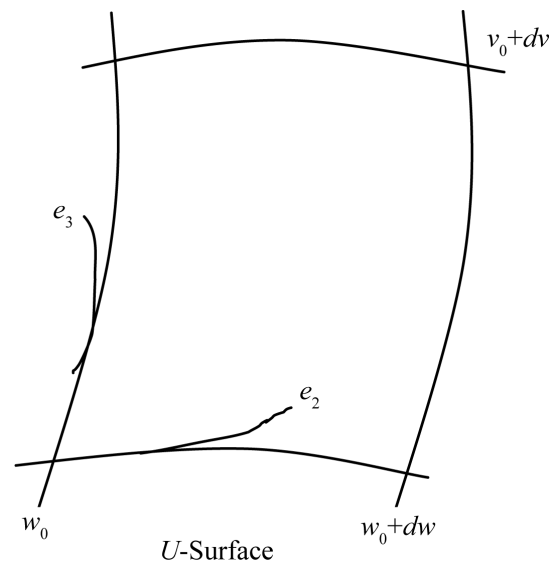

Figure 3. Infinitesimal region of space bounded by three coordinate surfaces. 
The infinitesimal region of space bounded by three coordinate surfaces has a volume given by

$$
\mathrm{d} v=\overline{\mathrm{d} S_{1}} \cdot\left(\overline{\mathrm{ds}_{2}} \times \overline{\mathrm{d} S_{3}}\right)=\overline{e_{1}} \cdot\left(\overline{e_{2}} \times \overline{e_{3}}\right) \mathrm{d} u \mathrm{~d} v \mathrm{~d} w .
$$

It follows from Equation (1.49) that the cross product $\bar{e}_{2} \times \bar{e}_{3}$ can be written as:

$$
\bar{e}_{2} \times \bar{e}_{3}=\left(\bar{e}_{1}^{-1} \bar{e}_{2} \times \bar{e}_{3}\right) \bar{e}_{1}+\left(\bar{e}_{2}^{-1} \bar{e}_{2} \times \bar{e}_{3}\right) \bar{e}_{2}+\left(\bar{e}_{3}^{-1} \bar{e}_{2} \times \bar{e}_{3}\right) \bar{e}_{3} .
$$

It follows from the definition of the reciprocal unitary vectors that we can rewrite equation (1.50) in the form:

$$
\bar{e}_{1} \cdot \bar{e}_{2} \times \bar{e}_{3}=\frac{\bar{e}^{-1}}{\bar{e}_{1} \bar{e}_{2} \times \bar{e}_{3}}\left[\left(\bar{e}_{2} \times \bar{e}_{3}\right) \cdot\left(\bar{e}_{1} \times \bar{e}_{2}\right) \bar{e}_{1}+\left(\bar{e}_{3} \times \bar{e}_{1}\right) \cdot\left(\bar{e}_{2} \times \bar{e}_{3}\right) \bar{e}_{2}+\left(\bar{e}_{1} \times \bar{e}_{2}\right) \cdot\left(\bar{e}_{3} \times \bar{e}_{1}\right) \bar{e}_{3}\right]
$$

which is equivalent to

$$
\begin{aligned}
\left(\bar{e}_{1} \cdot \bar{e}_{2} \times \bar{e}_{3}\right)^{2}= & \bar{e}_{1} \cdot \bar{e}_{1}\left[\left(\bar{e}_{2} \cdot \bar{e}_{2}\right) \cdot\left(\bar{e}_{3} \cdot \bar{e}_{3}\right)-\left(\bar{e}_{2} \cdot \bar{e}_{3}\right) \cdot\left(\bar{e}_{3} \cdot \bar{e}_{2}\right)\right]+\bar{e}_{1} \cdot \bar{e}_{2}\left[\left(\bar{e}_{2} \cdot \bar{e}_{3}\right) \cdot\left(\bar{e}_{3} \cdot \bar{e}_{1}\right)\right. \\
& \left.-\left(\bar{e}_{2} \cdot \bar{e}_{1}\right) \cdot\left(\bar{e}_{3} \cdot \bar{e}_{3}\right)\right]+\bar{e}_{1} \cdot \bar{e}_{3}\left[\left(\bar{e}_{2} \cdot \bar{e}_{1}\right) \cdot\left(\bar{e}_{3} \cdot \bar{e}_{2}\right)-\left(\bar{e}_{2} \cdot \bar{e}_{2}\right) \cdot\left(\bar{e}_{3} \cdot \bar{e}_{1}\right)\right] \\
= & g_{11}\left(g_{22} g_{33}-g_{23} g_{32}\right)+g_{22}\left(g_{23} g_{31}-g_{21} g_{33}\right)+g_{33}\left(g_{21} g_{32}-g_{22} g_{31}\right)
\end{aligned}
$$

The right hand side of Equation (1.51) is the expansion of the determinant

$$
g=\left|\begin{array}{lll}
g_{11} & g_{12} & g_{13} \\
g_{21} & g_{22} & g_{23} \\
g_{31} & g_{32} & g_{33}
\end{array}\right|
$$

Hence the increment volume is expressed in terms of the generalized coordinates $(u, v, w)$ by:

$$
\mathrm{d} V=\sqrt{g} \mathrm{~d} u \mathrm{~d} v \mathrm{~d} w .
$$

For the sake of simplicity, it will be convenient to make certain changes in notations and to introduce the so called Einstein summation convention. The change in notation which we require is the following:

$$
\begin{aligned}
& u=u^{1}, v=u^{2}, w=u^{3} \\
& x=x^{1}, y=x^{2}, z=x^{3}
\end{aligned}
$$

The summation convention which we shall adopt is the following. Whenever a Latin index appears both as a subscript and a superscript is the same expression, it is summed from 1 to 3 . For example:

$$
g_{i j}=g_{i_{1}} \mathrm{~d} u^{1}+g_{i_{2}} \mathrm{~d} u^{2}+g_{i_{3}} \mathrm{~d} u^{3}=\mathrm{d} u_{i} .
$$

In order to complete the convention, we shall adopt the rule that whenever an index appears as a subscript in the denominator of a fraction, it is regarded as equivalent to superscript in the numerator for purpose of summation and vice versa. For example:

$$
\frac{\partial u^{k}}{\partial x^{k}}=\frac{\partial u^{1}}{\partial x^{1}}+\frac{\partial u^{2}}{\partial x^{2}}+\frac{\partial u^{3}}{\partial x^{3}}
$$

\subsection{The Differential Operators in Generalized Coordinates}

The gradient of a scalar function $\rho\left(u^{1}, u^{2}, u^{3}\right)$ is a fixed vector which is defined to have the direction and magnitude of the maximum rate of change of $\rho$ with respect to the coordinates. The variation in $\rho$ corresponding to the infinitesimal displacement $\overline{\mathrm{dr}}$ is

$$
\mathrm{d} \rho=\overline{\mathrm{d} r} \cdot \operatorname{grad} \rho=\frac{\partial \rho}{\partial u^{i}} \mathrm{~d} u^{i} .
$$

Now the $\mathrm{d} u^{i}$ are the contra variant components of the infinitesimal displacement $\overline{\mathrm{d} r}$, and hence;

$$
\mathrm{d} u^{i}=\overline{\mathrm{d} r} \cdot \bar{e}_{i}^{-1} .
$$

Then 


$$
\left(\operatorname{grad} \rho-\frac{\partial \rho}{\partial u^{2}} \bar{e}_{i}^{-1}\right) \overline{\mathrm{d} r}=0 .
$$

Since the displacement $\overline{\mathrm{dr}}$ is arbitrary, it follows that the bracketed term in (1.59) must vanish identically. Thus the representation of gradient of the scalar field in terms of the generalized coordinates $\left(u^{1}, u^{2}, u^{3}\right)$ is

$$
\operatorname{grad} \rho=\frac{\partial \rho}{\partial u^{i}} \bar{e}_{i}^{-1} \text {. }
$$

The representation (1.60) is in terms of reciprocal unitary vectors, and hence, the quantities $\frac{\partial \rho}{\partial u^{i}}$ represent the covariant components of the gradient $\rho$. We may obtain a representation in terms of the unitary vectors from the relations:

$$
\bar{e}_{i}^{-1}=g^{i j} \bar{e}_{j}^{-1} .
$$

If we substitute these relations in (1.60), we obtain the representation

$$
\operatorname{grad} \rho=g^{i j} \frac{\partial \rho}{\partial u^{i}} .
$$

We then identify the contra variant components of $\operatorname{rrad} \rho$ as

$$
(\operatorname{grad} \rho)^{i}=g^{i j} \frac{\partial \rho}{\partial u^{j}} .
$$

The representation of the divergence of the vector field $\bar{a}\left(u^{1}, u^{2}, u^{3}\right)$ in terms of the generalized coordinates is easily obtained from the definition:

$$
\operatorname{div} \bar{a}\left(u^{1}, u^{2}, u^{3}\right)=\lim _{\nabla v \rightarrow 0} \frac{\int_{\nabla_{s}} \bar{a} \cdot \bar{n} \mathrm{dd} s}{\nabla v} .
$$

We shall evaluate the surface integral over the surface bounding the volume shown in Figure 4.

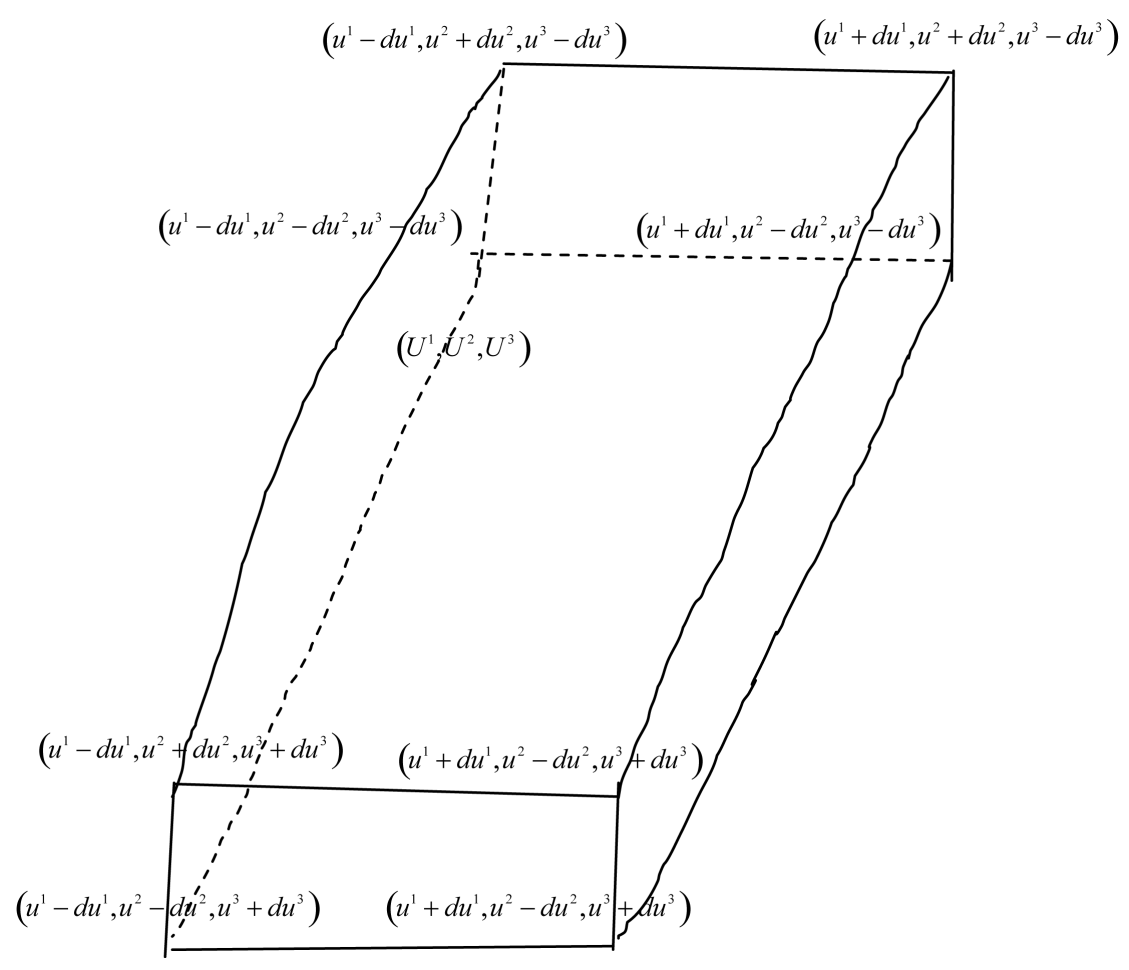

Figure 4. Surface bounding the volume $\bar{a}\left(u^{1}, u^{2}, u^{3}\right)$. 
The volume is bounded by the coordinate surface $u^{1}-\mathrm{d} u^{1}, u^{1}+\mathrm{d} u^{1}, u^{2}-\mathrm{d} u^{2}, u^{2}+\mathrm{d} u^{2}, u^{3}-\mathrm{d} u^{3}, u^{3}+\mathrm{d} u^{3}$ we consider the contribution to the surface integral from the two ends of the volume element which lie in $u^{1}$-surface. At the face $u^{1}+\mathrm{d} u^{1}$ we have:

$$
\bar{n} \mathrm{dd} s=4\left(\bar{e}_{2} \times \bar{e}_{2}\right) \mathrm{d} u^{2} \mathrm{~d} u^{2} \text {, while at the face } u^{1}-\mathrm{d} u^{1}, \bar{n} \mathrm{dd} s=4\left(\bar{e}_{3} \times \bar{e}_{2}\right) \mathrm{d} u^{2} \mathrm{~d} u^{3} .
$$

The contribution to the surface integral from these two faces is

$$
4\left[\bar{a} \cdot\left(\overline{e_{2}} \times \bar{e}_{3}\right) \mathrm{d} u^{2} \mathrm{~d} u^{3}\right]_{u^{1}+\mathrm{d} u^{1}}+4\left[\bar{a} \cdot\left(\overline{e_{3}} \times \bar{e}_{2}\right) \mathrm{d} u^{3} \mathrm{~d} u^{2}\right]_{u^{1}-\mathrm{d} u^{1}}
$$

where the subscript indicates that the quality in brackets is to be evaluated at the point indicated. The contribution to the surface integral from these two faces can be shown to be

$$
8 \frac{\partial}{\partial u^{1}}(a \sqrt{g}) \mathrm{d} u^{1} \mathrm{~d} u^{2} \mathrm{~d} u^{3} .
$$

These are analogous contributions from the remaining two pairs of faces. Adding, all the contributions to the surface integral dividing by

$$
\mathrm{d} v=8 \sqrt{g} \mathrm{~d} u^{1} \mathrm{~d} u^{2} \mathrm{~d} u^{3}
$$

and going to the limit $\mathrm{d} u^{1} \rightarrow 0, \mathrm{~d} u^{2} \rightarrow 0$ we obtain the desired representation

$$
\operatorname{div} \bar{a}=\frac{1}{\sqrt{g}} \frac{\partial}{\partial u^{i}}(a \sqrt{g}) .
$$

The representation of the curl of a vector field in terms of the set of generalized coordinates $\left(u^{1}, u^{2}, u^{3}\right)$ is also easily obtained from the definition

$$
\bar{n} \cdot \operatorname{curl} \bar{a}=\lim _{\Delta s \rightarrow 0} \frac{\int_{c} \bar{a} \cdot \mathrm{d} \bar{e}}{\Delta s}
$$

where $C$ is a closed contour bounding the surface $\Delta S$, which has unit positive normal $\bar{n}$. We shall evaluate the live integral about the contour. This bounds a rectangular region in the $u^{1}$-coordinate surface as in Figure 5 the direction of the contour has been chosen, so that the positive normal is in the direction of the position $u^{1}$-coordinate curve. The contribution to the live integral from the sides which are parallel to the $u^{2}$-coordinate curve is

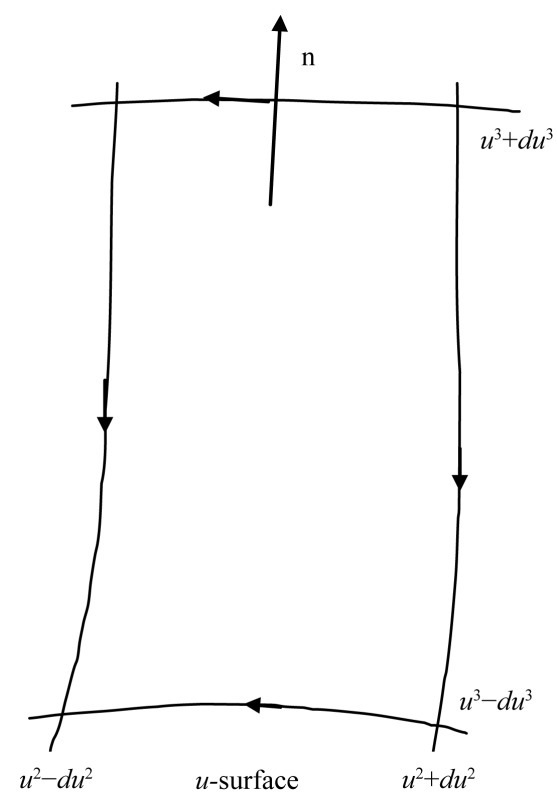

Figure 5. Rectangular region in $u$-coordinate surfaces. 


$$
-\left[\left(\bar{a} \cdot \bar{e}_{2}\right) \mathrm{d} u^{2}\right]_{u^{3}+\mathrm{d} u^{3}}+\left[\left(\bar{a} \cdot \bar{e}_{2}\right) \mathrm{d} u^{2}\right]_{u^{3}-\mathrm{d} u^{3}} .
$$

Similarly the sides parallel to the $u^{3}$-coordinate curves provide a contribution

$$
-\left[\left(\bar{a} \cdot \bar{e}_{3}\right) \mathrm{d} u^{3}\right]_{u^{2}+\mathrm{d} u^{2}}+\left[\left(\bar{a} \cdot \bar{e}_{3}\right) \mathrm{d} u^{3}\right]_{u^{2}-\mathrm{d} u^{2}} .
$$

Approximating Equations (1.68) and (1.69) by the linear terms of their Taylor series we find that the line integral is approximately by

$$
4\left[\frac{\partial}{\partial u^{2}}\left(\bar{a} \cdot \bar{e}_{3}\right)-\frac{\partial}{\partial u^{3}}\left(\bar{a} \cdot \bar{e}_{2}\right)\right] \mathrm{d} u^{2} \mathrm{~d} u^{3} .
$$

This quantity must now be divided by the area of the rectangular region which is

$$
\Delta S=4 \sqrt{\left\{\left(e_{2} \times e_{3}\right) \cdot\left(e_{2} \times e_{3}\right)\right\}} \mathrm{d} u^{2} \mathrm{~d} u^{3} .
$$

The reciprocal unitary vectors $\bar{e}^{-1}$ is always orthogonal to the $u^{1}$-coordinate surface, so that the unit must normal to the open surface is

$$
\bar{n}=\frac{\bar{e}^{-1}}{\sqrt{\left\{\bar{e}^{-1} \cdot \bar{e}^{-1}\right\}}}
$$

then

$$
(\operatorname{curl} \bar{a}) \bar{n}=(\operatorname{curl} \bar{a}) \frac{\bar{e}^{-1}}{\sqrt{\left\{\bar{e}^{-1} \cdot \bar{e}^{-1}\right\}}}=\frac{1}{\sqrt{\left\{\left(e_{2} \times e_{3}\right) \cdot\left(e_{2} \times e_{3}\right)\right\}}}\left[\frac{\partial}{\partial u^{2}}\left(\bar{a} \cdot \bar{e}_{3}\right)-\frac{\partial}{\partial u^{3}}\left(\bar{a} \cdot \bar{e}_{2}\right)\right]
$$

which will easily change to:

$$
\text { curl } \bar{a}=\frac{1}{\sqrt{g}}\left[\left(\frac{\partial a_{3}}{\partial u^{2}}-\frac{\partial a_{2}}{\partial u^{3}}\right) \bar{e}_{1}+\left(\frac{\partial a_{1}}{\partial u^{3}}-\frac{\partial a_{3}}{\partial u^{1}}\right) \bar{e}_{2}+\left(\frac{\partial a_{2}}{\partial u^{1}}-\frac{\partial a_{1}}{\partial u^{2}}\right) \bar{e}_{3}\right] .
$$

Equation (1.74) is the contra variant representation of $\operatorname{curl} \bar{a}$ with respect to the generalized coordinate system $\left(u^{1}, u^{2}, u^{3}\right)$.

\section{Orthogonal Coordinate System}

An orthogonal system is defined by the requirement that the unitary vectors $\left(e^{1}, e^{2}, e^{3}\right)$ are everywhere mutually orthogonal

$$
\bar{e}_{i}^{-1} \cdot \bar{e}_{j}=\bar{e}_{i j} \delta_{i j} .
$$

Under these conditions, the reciprocal unitary vector $\bar{e}^{-i}$ is parallel to $\bar{e}_{i}$ and is given by

$$
\bar{e}_{i}^{-1}=\frac{1}{\bar{e}_{i} \bar{e}_{j}} \cdot \bar{e}_{i}=\frac{1}{g_{i j}} \bar{e}_{i} .
$$

It follows from Equation (1.76) that the metric coefficients $g_{i j}$ vanish whenever $i \neq j$. For orthogonal coordinate system, it is necessary to define a set of scalar factors:

$$
h_{i}=\sqrt{g_{i j}} .
$$

In the case of an orthogonal system the set of second metric coefficients are given by:

$$
g^{i j}=\frac{1}{g_{i j}}=\frac{1}{h_{i}^{2}} \delta_{i j}
$$

from the sum of the first metric coefficients it follows that the scalar factors are calculated by

$$
h_{i}^{2}=\left(\frac{\partial x^{1}}{\partial u^{i}}\right)^{2}+\left(\frac{\partial x^{2}}{\partial u^{i}}\right)^{2}+\left(\frac{\partial x^{3}}{\partial u^{i}}\right)^{2}
$$


The element cell which is bounded by the coordinate surfaces is the rectangular region bounded by the edge of length

$$
\mathrm{d} S_{1}=h_{1} \mathrm{~d} u^{1}, \mathrm{~d} S_{2}=h_{2} \mathrm{~d} u^{2}, \mathrm{~d} S_{3}=h_{3} \mathrm{~d} u^{3} .
$$

The volume of this elementary cell is

$$
\mathrm{d} v=h_{1} h_{2} h_{3} \mathrm{~d} u^{1} \mathrm{~d} u^{2} \mathrm{~d} u^{3} .
$$

It is easy to see that thus is a special case of the previous result for the elementary volume ion a generalized coordinate system,

$$
\mathrm{d} v=\sqrt{g} \mathrm{~d} u^{1} \mathrm{~d} u^{2} \mathrm{~d} u^{3} .
$$

In the case of an orthogonal system, all of the off diagonal terms in $\operatorname{det}\left(g_{i j}\right)$ are zero, and consequently

$$
g=h_{1}^{2} h_{2}^{2} h_{3}^{2} .
$$

In a fixed coordinate system the advantages of using either covariant or contra variant components of a given vector $\bar{a}$, in terms of its physical components relative to the orthogonal unit base $\left(\bar{i}_{1}, \bar{i}_{2}, \bar{i}_{3}\right)$ where

$$
\bar{e}_{i}=h_{i} \overline{i_{i}}, \bar{e}^{i}=\frac{1}{h_{i}} \bar{i}_{i} .
$$

For an orthogonal system, we have the relatively simple relations between the covariant, contra variant and physical components of the vectors $\bar{a}$,

$$
a^{i}=\frac{1}{h_{i}} A_{i}, a^{i}=h_{i} A_{i} .
$$

The differential operator relative to an orthogonal curvilinear coordinate system can be immediately deduced as special cases of the general results. It follows from Equation (1.62) that in an orthogonal system, the gradient of a scalar field has the representation:

$$
\operatorname{grad} \phi=\sum_{j=1}^{3} \frac{1}{h_{j}} \frac{\partial \phi}{\partial u^{j}} \overline{i j}
$$

The divergence of a vector field in terms of an orthogonal curvilinear system can be obtained as special case of Equation (1.66).

$$
\operatorname{div} \bar{a}=\frac{1}{h_{1} h_{2} h_{3}}\left[\frac{\partial}{\partial u^{1}}\left(h_{2} h_{3} A_{1}\right)+\frac{\partial}{\partial u^{2}}\left(h_{3} h_{1} A_{2}\right)+\frac{\partial}{\partial u^{3}}\left(h_{1} h_{2} A_{3}\right)\right]
$$

Similarly, it follows from Equation (1.74) that is an orthogonal curvilinear system, the curl of a vector field has the representation

$$
\begin{aligned}
\text { curl } \bar{a}= & \frac{1}{h_{2} h_{3}}\left[\frac{\partial}{\partial u^{1}}\left(h_{3} A_{3}\right)-\frac{\partial}{\partial u^{3}}\left(h_{2} A_{2}\right)\right] \overline{i_{1}} \\
& +\frac{1}{h_{3} h_{1}}\left[\frac{\partial}{\partial u^{3}}\left(h_{3} A_{3}\right)-\frac{\partial}{\partial u^{1}}\left(h_{3} A_{3}\right)\right] \bar{i}_{2} \\
& +\frac{1}{h_{1} h_{2}}\left[\frac{\partial}{\partial u^{1}}\left(h_{2} A_{2}\right)-\frac{\partial}{\partial u^{2}}\left(h_{1} A_{1}\right)\right] \bar{i}_{3}
\end{aligned}
$$

In Equation (1.82) and (1.83), the quantities $A_{i}$ are the physical components of the vector $\bar{a}$ relative to the coordinate system $\left(u^{1}, u^{2}, u^{3}\right)$. We note that Equation (1.83) is the normal expansion of the determinant.

$$
\text { curl } \bar{a}=\frac{1}{h_{1} h_{2} h_{3}}\left|\begin{array}{ccc}
h_{1} \overline{i_{1}} & h_{2} \overline{i_{2}} & h_{3} \overline{i_{3}} \\
\frac{\partial}{\partial u^{1}} & \frac{\partial}{\partial u^{2}} & \frac{\partial}{\partial u^{3}} \\
h_{1} A_{1} & h_{2} A_{2} & h_{3} A_{3}
\end{array}\right|
$$




\section{Some Vector Relations in Curvilinear Coordinates}

In this section, we shall list the basic vector relations for two important curvilinear coordinate systems. We shall use the notation $(u, v, w)$ for the curvilinear systems, with the exception that in systems with cylinder symmetry, the coordinate axis along the symmetry axis will be denoted by $z$. The unit vector associated with the coordinate systems is specified in terms of their Cartesian representation, and all vector results are given in terms of physical components.

\subsection{Parabolic Cylindrical Coordinates}

$$
\begin{gathered}
x=(u-v) / 2, y=\sqrt{u v}, z=Z \\
0 \leq u<\infty, 0 \leq v<\infty,-\infty<z<\infty \\
h_{1}=\frac{1}{2} \sqrt{\frac{u+v}{u}}, h_{2}=\frac{1}{2} \sqrt{\frac{u+v}{v}}, h_{3}=1
\end{gathered}
$$

The constant coordinate surfaces are:

$$
\begin{aligned}
& u=\text { constant }- \text { parabolic cylinders } Y^{2}+2 u x=u^{2} \\
& v=\text { constant }- \text { parabolic cylinders } Y^{2}-2 v x=v^{2} \\
& z=\text { constant }- \text { planes normal to the cylinder axis }
\end{aligned}
$$

The unit vectors are

$$
\left.\begin{array}{c}
i_{1}=\sqrt{\frac{u}{u+v}} i+\sqrt{\frac{v}{u+v}} j \\
\left.i_{2}=-\sqrt{\frac{v}{u+v}} i+\sqrt{\frac{u}{u+v}} j\right\} \\
i_{3}=k
\end{array}\right\} \begin{gathered}
\operatorname{grad} \phi=\frac{2}{\sqrt{u+v}}\left(\sqrt{u} \frac{\partial \phi}{\partial u} i_{1}+\sqrt{v} \frac{\partial \phi}{\partial v} i_{2}\right)+\frac{\partial \phi}{\partial z} i_{3} \\
\operatorname{curl} \bar{A}=\frac{1}{\sqrt{u+v}}\left\{\left(2 \sqrt{\mathrm{A}}=\frac{2}{u+v}\left\{\sqrt{u} \frac{\partial}{\partial u}\left(\sqrt{(u+v)} A_{1}\right)+\sqrt{v} \frac{\partial}{\partial v}\left(\sqrt{(u+v)} A_{2}\right)\right\}+\frac{\partial A_{3}}{\partial z}-\sqrt{u+v} \frac{\partial A_{2}}{\partial z}\right) i_{1}+\left(\sqrt{u+v} \frac{\partial A_{1}}{\partial z}-2 \sqrt{u} \frac{\partial A_{3}}{\partial u}\right) i_{2}\right\} \\
+\frac{2}{u+v}\left[\sqrt{u} \frac{\partial}{\partial u}\left(\sqrt{(u+v)} A_{2}\right)-\sqrt{v} \frac{\partial}{\partial v}\left(\sqrt{(u+v)} A_{1}\right)\right] i_{3} \\
\nabla^{2} \phi=\frac{4}{u+v}\left\{\sqrt{u} \frac{\partial}{\partial u}\left(\sqrt{u} \frac{\partial \phi}{\partial u}\right)+\sqrt{v} \frac{\partial}{\partial v}\left(\sqrt{v} \frac{\partial \phi}{\partial v}\right)\right\}+\frac{\partial^{2} \phi}{\partial z^{2}}
\end{gathered}
$$

\subsection{Ellipsoidal Coordinates}

$$
x=\sqrt{\left\{\frac{\left(a^{2}-u\right)\left(a^{2}-v\right)\left(a^{2}-w\right)}{\left(a^{2}-b^{2}\right)\left(a^{2}-c^{2}\right)}\right\}}
$$




$$
\begin{gathered}
y=\sqrt{\left\{\frac{\left(b^{2}-u\right)\left(b^{2}-v\right)\left(b^{2}-w\right)}{\left(b^{2}-a^{2}\right)\left(c^{2}-b^{2}\right)}\right\}} \\
z=\sqrt{\left\{\frac{\left(c^{2}-u\right)\left(c^{2}-v\right)\left(c^{2}-w\right)}{\left(a^{2}-c^{2}\right)\left(b^{2}-c^{2}\right)}\right\}} \\
-\infty<u \leq c^{2} \leq v \leq b^{2} \leq w \leq a^{2} \\
h_{1}=\frac{1}{2} \sqrt{\left\{\frac{(v-u)(w-u)}{\left(a^{2}-u\right)\left(b^{2}-u\right)\left(c^{2}-u\right)}\right\}} \\
h_{2}=\frac{1}{2} \sqrt{\left\{\frac{(u-v)(w-v)}{\left(a^{2}-v\right)\left(b^{2}-v\right)\left(c^{2}-v\right)}\right\}} \\
h_{3}=\frac{1}{2} \sqrt{\left\{\frac{(u-w)(v-w)}{\left(a^{2}-w\right)\left(b^{2}-w\right)\left(c^{2}-w\right)}\right\}}
\end{gathered}
$$

The constant coordinate surfaces are

$$
\begin{aligned}
& u=\text { constant }- \text { ellipsoids } \frac{x^{2}}{a^{2}-u}+\frac{y^{2}}{b^{2}-u}+\frac{z^{2}}{c^{2}-u}=1 \\
& v=\text { constant }- \text { hyperboloids of one sheet } \frac{x^{2}}{a^{2}-v}+\frac{y^{2}}{b^{2}-v}+\frac{z^{2}}{c^{2}-v}=1 \\
& w=\text { constant }- \text { hyperboloids of two sheet } \frac{x^{2}}{a^{2}-w}+\frac{y^{2}}{b^{2}-w}+\frac{z^{2}}{c^{2}-w}=1
\end{aligned}
$$

The unit vectors are:

$$
\begin{aligned}
& i_{1}=\sqrt{\left\{\frac{\left(b^{2}-u\right)\left(c^{2}-u\right)\left(a^{2}-v\right)\left(a^{2}-w\right)}{(v-u)(w-u)\left(a^{2}-b^{2}\right)\left(a^{2}-c^{2}\right)}\right\}} i \\
& +\sqrt{\left\{\frac{\left(a^{2}-u\right)\left(c^{2}-u\right)\left(b^{2}-v\right)\left(b^{2}-w\right)}{(v-u)(w-u)\left(b^{2}-c^{2}\right)\left(a^{2}-b^{2}\right)}\right\}} j \\
& +\sqrt{\left\{\frac{\left(a^{2}-u\right)\left(b^{2}-u\right)\left(c^{2}-v\right)\left(c^{2}-w\right)}{(v-u)(w-u)\left(a^{2}-c^{2}\right)\left(b^{2}-c^{2}\right)}\right\}} k \\
& i_{2}=\sqrt{\left\{\frac{\left(b^{2}-v\right)\left(c^{2}-v\right)\left(a^{2}-u\right)\left(a^{2}-w\right)}{(u-v)(w-v)\left(a^{2}-b^{2}\right)\left(a^{2}-c^{2}\right)}\right\}} i \\
& +\sqrt{\left\{\frac{\left(a^{2}-v\right)\left(c^{2}-v\right)\left(b^{2}-u\right)\left(b^{2}-w\right)}{(u-v)(w-v)\left(b^{2}-c^{2}\right)\left(a^{2}-b^{2}\right)}\right\}} j
\end{aligned}
$$

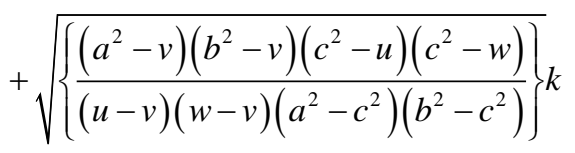




$$
\begin{aligned}
& i_{3}=\sqrt{\left\{\frac{\left(b^{2}-w\right)\left(c^{2}-w\right)\left(a^{2}-u\right)\left(a^{2}-v\right)}{(u-w)(v-w)\left(a^{2}-b^{2}\right)\left(a^{2}-c^{2}\right)}\right\}} i \\
& +\sqrt{\left\{\frac{\left(a^{2}-w\right)\left(c^{2}-w\right)\left(b^{2}-u\right)\left(b^{2}-v\right)}{(u-w)(v-w)\left(b^{2}-c^{2}\right)\left(a^{2}-b^{2}\right)}\right\}} j \\
& +\sqrt{\left\{\frac{\left(a^{2}-w\right)\left(b^{2}-w\right)\left(c^{2}-u\right)\left(c^{2}-v\right)}{(u-w)(v-w)\left(a^{2}-c^{2}\right)\left(b^{2}-c^{2}\right)}\right\} k} \\
& \operatorname{grad} \phi=\frac{2 \sqrt{\left\{\left(a^{2}-u\right)\left(b^{2}-u\right)\left(c^{2}-u\right)\right\}}}{(v-u)(w-u)} \frac{\partial \phi}{\partial u} i_{1} \\
& +\frac{2 \sqrt{\left\{\left(a^{2}-v\right)\left(b^{2}-v\right)\left(c^{2}-v\right)\right\}}}{(u-v)(v-w)} \frac{\partial \phi}{\partial v} i_{2} \\
& +\frac{2 \sqrt{\left\{\left(a^{2}-w\right)\left(b^{2}-w\right)\left(c^{2}-w\right)\right\}}}{(u-w)(v-w)} \frac{\partial \phi}{\partial w} i_{3} \\
& \operatorname{div} \bar{A}=\frac{2 \sqrt{\left\{\left(a^{2}-u\right)\left(b^{2}-u\right)\left(c^{2}-u\right)\right\}}}{(v-u)(w-u)} \frac{\partial}{\partial u}\left[\sqrt{\{(v-u)(u-w)\}} A_{1}\right] \\
& +\frac{2 \sqrt{\left\{\left(a^{2}-v\right)\left(b^{2}-v\right)\left(c^{2}-v\right)\right\}}}{(v-u)(v-w)} \frac{\partial}{\partial v}\left[\sqrt{\{(v-u)(w-v)\}} A_{2}\right] \\
& +\frac{2 \sqrt{\left\{\left(a^{2}-w\right)\left(b^{2}-w\right)\left(c^{2}-w\right)\right\}}}{(v-w)(w-u)} \frac{\partial}{\partial w}\left[\sqrt{\{(w-v)(u-w)\}} A_{3}\right] \\
& \operatorname{curl} \bar{A}=\frac{2}{v-w} \sqrt{\left\{\frac{\left(a^{2}-v\right)\left(b^{2}-v\right)\left(c^{2}-v\right)}{v-u}\right\}} \frac{\partial}{\partial v}\left[\sqrt{(v-w)} A_{3}\right] \\
& -\sqrt{\left\{\frac{\left(a^{2}-w\right)\left(b^{2}-w\right)\left(c^{2}-w\right)}{w-u}\right\}} \frac{\partial}{\partial w}\left[\sqrt{(w-v)} A_{2}\right] i_{1} \\
& +\frac{2}{w-u} \sqrt{\left\{\frac{\left(a^{2}-w\right)\left(b^{2}-w\right)\left(c^{2}-w\right)}{w-v}\right\}} \frac{\partial}{\partial w}\left[\sqrt{(w-u)} A_{1}\right] \\
& -\sqrt{\left\{\frac{\left(a^{2}-u\right)\left(b^{2}-u\right)\left(c^{2}-u\right)}{u-v}\right\}} \frac{\partial}{\partial u}\left[\sqrt{(u-w)} A_{3}\right] i_{2} \\
& +\frac{2}{u-v} \sqrt{\left\{\frac{\left(a^{2}-u\right)\left(b^{2}-u\right)\left(c^{2}-u\right)}{v-w}\right\}} \frac{\partial}{\partial u}\left[\sqrt{(u-v)} A_{2}\right] \\
& \left.-\sqrt{\left\{\frac{\left(a^{2}-v\right)\left(b^{2}-v\right)\left(c^{2}-v\right)}{v-w}\right\}}\right\} \frac{\partial}{\partial v}\left[\sqrt{(v-u)} A_{1}\right] i_{3}
\end{aligned}
$$




$$
\begin{aligned}
\nabla^{2} \phi= & \frac{4 \sqrt{\left\{\left(a^{2}-u\right)\left(b^{2}-u\right)\left(c^{2}-u\right)\right\}}}{(v-u)(w-u)} \frac{\partial}{\partial u}\left[\sqrt{\left\{\left(a^{2}-u\right)\left(b^{2}-u\right)\left(c^{2}-u\right)\right\}} \frac{\partial \phi}{\partial u}\right] \\
& +\frac{4 \sqrt{\left\{\left(a^{2}-v\right)\left(b^{2}-v\right)\left(c^{2}-v\right)\right\}}}{(u-v)(w-v)} \frac{\partial}{\partial v}\left[\sqrt{\left\{\left(a^{2}-v\right)\left(b^{2}-v\right)\left(c^{2}-v\right)\right\}} \frac{\partial \phi}{\partial v}\right] \\
& +\frac{4 \sqrt{\left\{\left(a^{2}-w\right)\left(b^{2}-w\right)\left(c^{2}-w\right)\right\}}}{(u-w)(v-w)} \frac{\partial}{\partial w}\left[\sqrt{\left\{\left(a^{2}-w\right)\left(b^{2}-w\right)\left(c^{2}-w\right)\right\}} \frac{\partial \phi}{\partial w}\right]
\end{aligned}
$$

\section{Conclusion}

A short discussion on curvilinear coordinates has been given. We specialize here the general results of the case of orthogonal systems. We have also given different vector results in two orthogonal coordinates systems, viz. parabolic cylindrical and ellipsoidal system of coordinates.

\section{References}

[1] Ballabh, R. (1952) Two Dimensional Superposable Motions. Journal of the Indian Mathematical Society, 16, $191-197$.

[2] Bhatia, B.L. (1971) Continuity Condition in Parabolic Cylinder System. The Mathematics Student, 39, 167-180.

[3] Gupta, S. and Rajan, S. (2007) On Some Magnetohydrodynamics Configuration in Parabolic Cylindical Ducts. Acta Ciencia Indica, XXXIIIM, 919-926.

[4] Mittal, P.K., Singh, V. and Rajan, S. (1997) A Note on Vorticity of Hydromagnetic Converting Slip Flow through a Horizontal Channel. Acta Ciencia Indica, XXIIIM, 117.

[5] Mittal, P.K. (1983) On Some Magnetohydrostatic Configurations in Parabolic Coordinated. Bulletin of Calcutta Mathematical Society, 75, 339-352.

[6] Mittal, P.K. (1986) On Some Self-Superposable Fluid Motions in Paraboloidal Ducts. International Journal of Theoretical Physics, 34, 181-191.

[7] Mittal, P.K. and Khan, M.I. (1986) On Some Self-Superposable Flows in Conical Ducts. Jhanabha, 16, 91-102.

[8] Mittal, P.K., Thapaliyal, P.S. and Khan, M.I. (1987) On Some Magnetohydrodynamics Configurations in Conical Ducts. Indian Journal of Physics and Natural Science, 8B, 7-14.

[9] Mittal, P.K., Chandra, S. and Salam, S.B. (1987) On Some Magnetohydrodynamics Configuration in Parabolic Ducts. Indian Journal of Physics and Natural Science, 8B, 48-53.

[10] Ballabh, R. (1963) U.P. Scientific Committee Monograph 1963.

[11] Truesdall, C. (1954) The Kinematics of Vorticity. Indiana University Press, Bloomington.

[12] Gold, R.R. and von Krzywoblocki, M.Z. (1958) On Superposability and Self-Superposability Conditions for Hydrodynamic Equations Based on Continuum. I. Journal für die reine und angewandte Mathematik, 199, 139-164.

[13] Gold, R.R. and von Krzywoblocki, M.Z. (1958) On Superposability and Self-Superposability Conditions for Hydrodynamic Equations Based on Continuum. II. Journal für die reine und angewandte Mathematik, 200, 140-169.

[14] Gupta, S. and Rajan, S. (2007) Rotating Disk Flow with Heat Transfer of a Non-Newtonian Fluid in Porous Medium. Acta Ciencia Indica, XXXIIIM, 1031-1036.

[15] Kapur, J.N. and Bhatia, B.L. (1965) Superposability and Self-Superposability in Fluiddynamics-II. Proceedings of the National Institute of Sciences of India, 31A, 126-151.

[16] Kapur, J.N. (1959) Superposability in Magnetohydrodynamics. Applied Scientific Research, 8, 198-208.

[17] Kapur, J.N. (1960) Superposability in Magnetohydrodynamics II. Applied Scientific Research, 9, 139-147.

[18] Bhatnagar, P.L. (1960) Superposability and Harmonic Analysis of Flows of a Viscous Liquid in the Presence of Magnetic Field. Jubilee Commemoration Volume of the Calcutta Mathematical Society, 205-216.

[19] Kapur, J.N. (1961) Superposability and Self-Superposability in Fluid Dynamics. The Mathematics Seminar, 2, 1-31.

[20] Kapur, J.N. (1962) Some Properties of Force-Free Fields. The Mathematics Seminar, 2, 135-138.

[21] Kapur, J.N. (1962) Characterisation of Axially-Symmetric Self-Superposable Flows in Magnetohydrodynamic. Bulletin of Calcutta Mathematical Society, 59-66. 
[22] Chandrasekhar, S. (1981) Hydrodynamics and Hydromagnetics Stability. Dover Publication, New York.

[23] Rao, G.T. (1960) Superposability of the Equations of Magneto-Hydrodynamics. Journal of the Mathematical Society of Japan, 12, 97-103.

[24] Ramamoorthy, P. (1960) Superposability of Two Axi-Symmetric Flows under Axi-Symmetric Magnetic Fields. Applied Scientific Research, 9, 153-156.

[25] Mittal, P.K. (1977) A Note on Vorticity in Conducting Fluid. The Mathematics Student, 45, 84-88.

[26] Mittal, P.K. (1981) A Note on Steady Laminar Magneto Hydrodynamic Flow. Bulletin of Calcutta Mathematical Society, 73, 179-184.

[27] Mittal, P.K. (1978) On the Vorticity of the MHD Flow in a Rectangular Duct. The Mathematics Education, 12, 5-9.

[28] Mittal, P.K., Thapaliyal, P.S. and Aarwal, G.K. (1987) On Some Self-Superposable Fluid Motions in Toroidal Ducts. Proceedings of the National Academy of Sciences, India, Section A, 57, 224-229.

[29] Mittal, P.K. and Rastogi, S.C. (1987) Self-Superposable Motions in Paraboloidal Ducts. Indian Journal of Physics and Natural Science, 8B, 27-32.

[30] Mittal, P.K., Negi, B.S. and Shamshi, S.R. (1986) Self-Superposable Flows in Ducts having Confocal Ellipsoidal Shape. Indian Journal of Physics and Natural Science, 7B, 1-10.

[31] Rastogi, S., Kaul, B.N. and Rajan, S. (2015) Few Magnetohydrostatic Forms in Confocal Paraboloidal Ducts. International Journal of Mathematics and Computer Application Research, 5, 25-34.

[32] Singh, V. and Rajan, S. (1997) On Some Hydrodynamic Flows in Ellipsoidal Ducts. Acta Ciencia Indica, XXXIIIM, 111. 